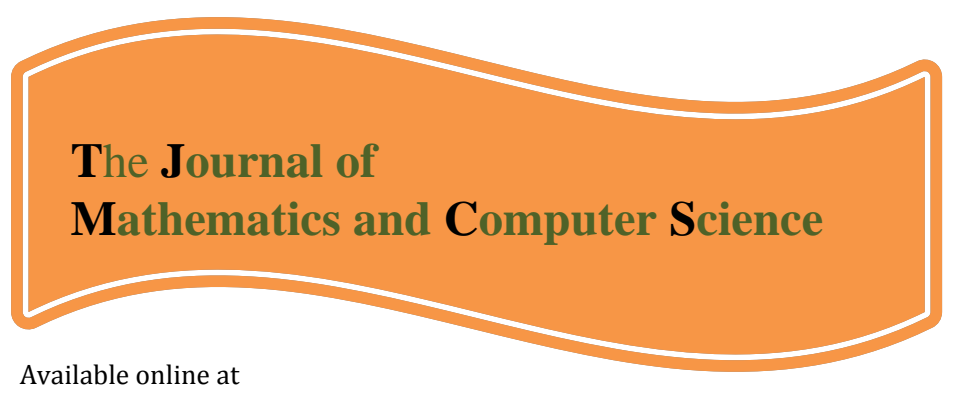

http://www.TIMCS.com

The Journal of Mathematics and Computer Science Vol .2 No.2 (2011) 388-393

\title{
Variational iteration method: A tools for solving partial differential equations
}

\author{
Elham Salehpoor ${ }^{a}$ Hossein Jafari ${ }^{b}$ \\ aDepartment of Mathematics, Islamic Azad University Nooshahr Branch, \\ Nooshahr, Iran \\ elham_salehpor61@yahoo.com \\ bDepartment of Mathematics, University of Mazandran, Babolsar, Iran \\ Jafari@umz.ac.ir
}

Received: August 2010, Revised: November 2010

Online Publication: January 2011

\section{Abstract}

In this paper, He's variational iteration method (VIM) has been used to obtain solution nonlinear gas dynamics equation and Stefan equation. This method is based on Lagrange multipliers for identification of optimal values of parameters in a functional. Using this method creates a sequence which tends to the exact solution of the problem.

Keywords

Variational iteration method; nonlinear gas dynamics equation; Stefan equation; Partial differential equation.

\section{Introduction}

Analytical methods commonly used to solve nonlinear equations are very restricted and numerical techniques involving discretization of the variables on the other hand gives rise to rounding off errors.

Recently introduced variational iteration method by $\mathrm{He}[5,6,7,8]$, which gives rapidly convergent successive approximations of the exact solution if such a 
solution exists, has proved successful in deriving analytical solutions of linear and nonlinear differential equations. This method is preferable over numerical methods as it is free from rounding off errors and neither requires large computer power/memory. He [6,7] has applied this method for obtaining analytical solutions of autonomous ordinary differential equation, nonlinear partial differential equations with variable coefficients and integro-differential equations. The variational iteration method was successfully applied to Seventh order Sawada-Kotera equations [11], to Schruodinger-KdV, generalized KdV and shallow water equations [1], to linear Helmholtz partial differential equation [12]. Linear and nonlinear wave equations, $\mathrm{KdV}, \mathrm{K}(2,2)$, Burgers, and cubic Boussinesq equations have been solved by Wazwaz $[14,15]$ using the variational iteration method. In the present paper we employ VIM method for solving following equations. Consider the homogeneous gas dynamics equation

$\frac{\partial u}{\partial t}+\frac{1}{2} \frac{\partial u^{2}}{\partial x}=u(1-u), \quad 0 \leq \mathrm{x} \leq 1, \mathrm{t} \geq 0$

with initial condition

$u(x, 0)=g(x)$.

Also we employ VIM method for solving following equations. Consider the Stefan equation

$\frac{\partial u}{\partial t}=\frac{\partial u^{2}}{\partial x^{2}}, \quad 0 \leq \mathrm{x} \leq \mathrm{s}(t), \mathrm{t} \geq 0$

with initial condition

$u(x, 0)=-1, \quad \mathrm{t} \geq 0$

$u(s(x), t)=0, \quad \mathrm{t} \geq 0$

$\frac{\partial u}{\partial x}=\frac{\partial s(x)}{\partial t}$.

Further we compare the result with given solutions using ADM $[2,3,4]$. The paper has been organized as follows. Section 2, gives a brief review of VIM. Section 3,5 consists of main results of the paper, in which variational iteration method of nonlinear gas dynamics equation and nonlinear Stefan equation have been developed. In Section 4,6 illustrative examples are given.In Section 6,illustrative examples are given. Conclusions are presented in Section 7.

\section{He's variational iteration method}

According to the variational iteration method, we consider the following differential equation:

$L u+N u=g(x, t)$,

Where $L$ is a linear operator, $N$ a non-linear operator and $g(t)$ is the source inhomogeneous term. According to the variational interation method, we can construct a Correction functional as follow

$u_{n+1}(x, t)=u_{n}(x, t)+\int_{o}^{t} \lambda(\xi)\left\{L u_{n}(\xi)+N \tilde{u}_{n}(\xi)-g(\xi)\right\} d \xi, \mathrm{n} \geq 0$, 
Where $\lambda$ is a general Lagrangian multiplier, which can be identified optimally via the variational theory, the second term on the right is called the correction and $\tilde{u}_{n}$ is considered as a restricted variation, i.e., $\delta \tilde{u}_{n}=0$.

So, we first determine the Lagrange multiplier $\lambda$ that will be identified optimally via integration by parts. The successive approximations $u_{n+1}(x, t), n \geq 0$ of the solution $u(x, t)$ will be readily obtained upon using the obtained Lagrange multiplier and by using any selective function $u_{0}$.Consequently, the solution

$\mathbf{u}(\mathbf{x}, \mathbf{t})=\lim _{\mathbf{n} \rightarrow \infty} \mathbf{u}_{\mathbf{n}}(\mathbf{x}, \mathbf{t})$.

\section{Applying VIM for nonlinear gas dynamics equation}

In this section, we shall introduce a reliable algorithm based on VIM to handle singular initial value problem in a realistic and efficient way considering gas dynamics equation as a model problem.

Its correction variational functional in t-direction to obtain the solution of gas dynamics equation (1) by variational iteration method can be expressed as follows:

$u_{n+1}(x, t)=u_{n}(x, t)+\int_{0}^{t} \lambda(\xi)\left[\frac{\partial}{\partial \xi}\left(u_{n}\right)-u_{n}+\left(N\left(\tilde{u}_{n}\right)\right)\right] d \xi, \mathrm{n} \geq 0$

where $N \tilde{u}_{n}(x, \xi)=\frac{1}{2} \tilde{u}_{n x}^{2}(x, \xi)+\tilde{u}^{2}(x, \xi)$.taking variation with respect to the independent variable $u_{n}$ noticing that $\delta N \tilde{u}_{n}(x, \xi)=0$.

$$
\begin{aligned}
\delta u_{n+1}(x, t) & =\delta u_{n}(x, t)+\delta \int_{0}^{t} \lambda(\xi)\left[\frac{\partial}{\partial \xi}\left(u_{n}\right)-u_{n}+\left(N\left(\tilde{u}_{n}\right)\right)\right] d \xi \\
& =\delta u_{n}(x, t)+\left.\lambda \delta u_{n}\right|_{\xi=t}-\int_{0}^{t} \lambda^{\prime}(\xi) \delta u_{n}+\lambda(\xi) \delta u_{n} d \xi=0,
\end{aligned}
$$

This yields the stationary conditions

$$
1+\lambda(\xi)=0, \quad \lambda(\xi)+\left.\lambda^{\prime}(\xi)\right|_{\xi=t}=0 .
$$

This in turn gives $\lambda(\xi)=-\mathrm{e}^{\mathrm{t}-\xi}$. Substituting this value of the Lagrange multiplier into the functional (13)gives the iteration formula

$u_{n+1}(x, t)=u_{n}(x, t)+\int_{0}^{t} e^{t-\xi}\left[\frac{\partial}{\partial \xi}\left(u_{n}\right)-u_{n}+\left(N\left(\tilde{u}_{n}\right)\right)\right] d \xi, \quad \mathrm{n} \geq 0$

thus, we can obtain approximation solutions for $\mathrm{u}(\mathrm{x}, \mathrm{t})$, considering boundary conditions and insert $u(x, t) \cong u_{n}(x, t)$.

\section{Illustrative Example}

To demonstrate the effectiveness of the method we consider here Eqs. [3] with given initial condition.

Example 4.1 Consider the nonlinear gas dynamics equations (1) with the initial condition 
$u(x, 0) \cong e^{-x}$.

Substituting(11) into Eq.(10) we obtain the following successive approximations

$$
\begin{aligned}
u_{0}(x, t) & \cong e^{-x}, \\
u_{1}(x, t) & =u_{0}(x, t)-\int_{0}^{\mathrm{t}} e^{t-\xi}\left[\frac{\partial}{\partial \xi}\left(u_{0}\right)-u_{0}+\frac{1}{2} \tilde{u}_{0 x}^{2}(x, \xi)+\tilde{u}_{0}^{2}(x, \xi)\right] d \xi, \\
& =\mathrm{e}^{\mathrm{t}-\mathrm{x}}, \\
u_{2}(x, t) & =u_{1}(x, t)-\int_{0}^{\mathrm{t}} e^{t-\xi}\left[\frac{\partial}{\partial \xi}\left(u_{1}\right)-u_{1}+\frac{1}{2} \tilde{u}_{1 x}^{2}(x, \xi)+\tilde{u}_{1}^{2}(x, \xi)\right] d \xi, \\
& =\mathrm{e}^{\mathrm{t}-\mathrm{x}},
\end{aligned}
$$

Finally, $u_{n}(x, t)=\mathrm{e}^{\mathrm{t}-\mathrm{x}}$, then we can write, $u(x, t)=\mathrm{e}^{\mathrm{t}-\mathrm{x}}$.

we can see that calculated solution will be the following exact solution:

$u(x, t)=\mathrm{e}^{\mathrm{t}-\mathrm{x}}$

Remark 1: Nonlinear gas dynamics equation has been solved by ADM by Evans and Bulut [3]. they obtained exact solution after some iteration but we obtain exact solution after 2 iteration.

\section{Applying VIM for Stefan problem}

In this section, we apply VIM to stefan prablem(Eq.(3)). Its correction variational functional in t-direction to obtain the solution of stefan prablem (3) by variational iteration method can be expressed as follows:

$u_{n+1}(x, t)=u_{n}(x, t)+\int_{0}^{x} \lambda(\xi, t)\left[\frac{\partial^{2}}{\partial \xi^{2}}\left(u_{n}\right)+\left(N\left(\tilde{u}_{n}\right)\right)\right] d \xi, \mathrm{n} \geq 0$,

where $N \tilde{u}_{n}(\xi, t)=-\tilde{u}_{n t}(\xi, t)$. taking variation with respect to the independent variable $u_{n}$ noticing that $\delta N \tilde{u}_{n}(\xi, t)=0$

$$
\begin{aligned}
\delta u_{n+1}(x, t) & =\delta u_{n}(x, t)+\delta \int_{0}^{x} \lambda(\xi, t)\left[\frac{\partial^{2}}{\partial \xi^{2}}\left(u_{n}\right)+\left(N\left(\tilde{u}_{n}\right)\right)\right] d \xi \\
& =\delta u_{n}(x, t)+\left.\lambda(\xi, t) \delta u_{n x}\right|_{\xi=x} \\
& -\left.\lambda^{\prime}(\xi, t) \delta u_{n}\right|_{\xi=x}+\int_{0}^{x} \lambda^{\prime \prime}(\xi, t) \delta u_{n} d \xi=0
\end{aligned}
$$

This yields the stationary conditions

$1-\lambda^{\prime}(\xi, t)_{\xi=x}=0, \quad \lambda(\xi, t)_{\xi=x}=0, \quad \lambda^{\prime \prime}(\xi, t)_{\xi=x}=0$.

This in turn gives $\lambda(\xi, t)_{\xi=x}=\xi-x$. Substituting this value of the Lagrange multiplier into the functional[13] gives the iteration formula

$u_{n+1}(x, t)=u_{n}(x, t)+\int_{0}^{\mathrm{x}}(\xi-x)\left[\frac{\partial^{2}}{\partial \xi^{2}}\left(u_{n}\right)+\left(N\left(\tilde{u}_{n}\right)\right)\right] d \xi$,

thus, we can obtain approximation or exact solution for $u(x, t)$.

\section{Illustrative Example}


To demonstrate the effectiveness of the method we consider here Eqs. [3] with given initial condition.

Example 6.1 Consider Stefan problem (3) with the I.C. $u_{0}(x, t)=-x+e^{-t}$.

Substituting [18] into [17] we obtain the following successive approximations

$$
\begin{aligned}
& u_{1}(x, t)=-x+e^{t}+\frac{1}{2} x^{2} e^{t}, \\
& u_{2}(x, t)=-x+e^{t}+\frac{1}{2} x^{2} e^{t}+\frac{1}{24} x^{4} e^{t}, \\
& u_{3}(x, t)=-x+e^{t}+\frac{1}{2} x^{2} e^{t}+\frac{1}{24} x^{4} e^{t}+\frac{1}{720} x^{6} e^{t},
\end{aligned}
$$

finally, $u_{n}(x, t)=-x+e^{t} \cosh x$, we have $u(x, t)=-x+e^{t} \cosh x$ which is exact solution.

\section{Conclusion}

Variational iteration method is a powerful tool which is capable of handling linear/nonlinear partial differential equations. The method have been successfully applied to nonlinear gas dynamics equation and Stefan equation.

Also, comparisons were made between He's variational iteration method and Adomian decomposition method (ADM) for nonlinear gas dynamics equation and Stefan equation.

The VIM reduces the volume of calculations without requiring to compute the Adomian polynomials. He's variational iteration method facilitates the computational work and gives the solution rapidly if compared with Adomian method.

\section{References}

[1] M.A. Abdou, A.A. Soliman, New applications of variational iteration method, Physica D, 211 (2005) 1-8.

[2] G. Adomian, Solving Frontier Problems of Physics: The Decomposition Method, Kluwer, 1994.

[3] D.J. Evans and H.Bulut,A new approach to the gas dynamics equation:An application of the decomposition method, Appl.Comput.Math.79 (7)(2002) 817-822.

[4] R.Grzymkowski and D,Slota, Stefan problem solved by Adomian decomposition method, Chaos, Solitons and Fractals, 82 (7) (2005) 851-856.

[5] J.H. He, Variational iteration method -Some recent results and new interpretations, J. Comput. Intern. J. Math., 207 (1) (2007) 3-17. 
[6] J.H. He, Variational principles for some nonlinear partial differential equations with variable coefficients, Chaos, Solitons and Fractals, 19 (2004) 847-851.

[7] J. H. He, Variational iteration method for autonomous ordinary differential systems, Appl. Math. Comput., 114 (2000) 115-123.

[8] J. H. He, Some asymptotic methods for strongly nonlinear equations, Int. J. Mod. Phys. B, 20 (10) (2006) 1141-1199.

[9] W. Hereman, P.P. Banerjee, A. Korpel, G. Assanto, A. van Immerzeele and A. Meerpoel, Exact solitary wave solutions of nonlinear evolution and wave equations using a direct algebraic method, J. Phys. A: Math. Gen., 19 (1986) 607-628.

[10] M. Inokuti, et al., General use of the Lagrange multiplier in non-linear mathematical physics, in: S. Nemat-Nasser (Ed.), Variational Method in the Mechanics of Solids, Pergamon Press, Oxford, 1978, pp. 156-162.

[11] H. Jafari, et al., Application of He's Variational Iteration Method for Solving Seventh Order Sawada-Kotera Equations, Applied Mathematical Sciences, 2 (10), 2008, 471 - 477.

[12] S. Momani and S. Abuasad, Application of He's variational iteration method to Helmholtz equation, Chaocs, Solitons and Fractals, 27 (2006) 1119-1123.

[13] E. J. Parkes and B.R. Duffy, An automated tanh-function method for finding solitary wave solutions to non-linear evolution equations, Comput. Phys. Commun., 98 (1996) 288-300.

[14] A. M. Wazwaz, The variational iteration method: A reliable analytic tool for solving linear and nonlinear wave equations, Computers and Mathematics with Applications, 54 (2007) 926-932.

[15] A. M. Wazwaz, The variational iteration method for rational solutions for $\mathrm{KdV}, \mathrm{K}(2,2)$, Burgers, and cubic Boussinesq equations, J. Comput. Appl. Math., 207 (1) (2007) 18-23. 